\title{
An Efficient Intragenic Vector for Generating Intragenic and Cisgenic Plants in Citrus
}

\author{
Chuanfu An ${ }^{1}$, Vladimir Orbović ${ }^{2}$, Zhonglin $\mathrm{Mou}^{{ }^{*}}$ \\ ${ }^{1}$ Department of Microbiology and Cell Science, University of Florida, Gainesville, USA; ${ }^{2}$ Citrus Research and Education Center, \\ University of Florida, Lake Alfred, USA. \\ Email: zhlmou@ufl.edu
}

Received September $2^{\text {nd }}, 2013$; revised October $2^{\text {nd }}, 2013$; accepted October $23^{\text {rd }}, 2013$

Copyright (C) 2013 Chuanfu An et al. This is an open access article distributed under the Creative Commons Attribution License, which permits unrestricted use, distribution, and reproduction in any medium, provided the original work is properly cited.

\begin{abstract}
Genetic transformation has become a promising tool for improvement of a variety of crop species. However, transferring genes across species, the presence of selectable marker genes, and bacteria-derived vector backbone sequences have raised considerable health and environmental concerns. Intragenic vector system-based intragenesis/cisgenesis is a new method using transgenic approach to achieving traditional breeding objectives but circumventing many of the associated shortcomings. We report here the development of an intragenic vector by assembling a T-DNA-like fragment and a buffering sequence following the left border from Citrus clementina into the backbone of the binary vector pCB302. Recovery of citrus regenerants is performed under non-selective conditions and positive intra-/cisgenic regenerants were identified through PCR analysis. Transformation efficiencies obtained in Arabidopsis and "Duncan" grapefruit were $\sim 3 \%$ and $\sim 0.67 \%$, respectively, demonstrating the potential of the system for development of "foreign DNA-free" intra-/cisgenic citrus cultivars.
\end{abstract}

Keywords: Intragenesis/Cisgenesis; Citrus; Selectable Marker; Transgenesis

\section{Introduction}

Genetic improvement of citrus (Citrus spp.) by conventional breeding is hindered by incompatibility, apomixes, heterozygosity, and lengthy juvenile period. Transgenesis has become a promising tool to directly introduce desirable traits into elite genotypes without altering existing genetic background. However, the release of genetically modified (GM) crops has raised considerable health and environmental concerns mainly due to the transferring of genes across wide taxonomic boundaries and the presence of selectable marker genes [1,2]. With the accumulating knowledge of the structure and function of genes from crop species, delivering useful alleles of native genes into crossable plant species or genotypes has become possible. In addition, a number of strategies have been developed to remove marker genes from transgenic plants $[3,4]$. However, currently preferred Agrobacterium-mediated plant genetic transformation and elimination of selectable marker genes largely rely on prokaryotederived vector systems [5]. With this method, foreign

${ }^{*}$ Corresponding author. genes or sequences still remain in the GM plants.

Intragenic vector system-based intra-/cisgenesis combines the benefits of traditional breeding and genetic engineering, but circumvents many of their problematic issues [5,6]. It involves identifying functional equivalents of vector components from target or crossable plant species and using these DNA sequences to assemble vector for plant transformation. Native genes from a sexually compatible species can be delivered into elite cultivars by the intragenic vector system in a single step without linkage drag and, most importantly, without the incorporation of "foreign DNA" [6].

In citrus, although there is a report on recovering selectable marker-free transgenic orange plant under non-selective conditions, no intra-/cisgenesis experiment has been conducted due to the lack of an intragenic vector system [7]. We present here the development of a "foreign DNA-free" intragenic vector system, pUFCI (University of Florida Citrus Intragenic), by assembling T-DNA-like fragments with functional equivalents of T-DNA border sequences from C. clementina and recovery of positive intra-/cisgenic regenerants through PCR 
analysis. The transformation efficiencies obtained in both Arabidopsis and "Duncan" grapefruit indicate its great potential for citrus genetic improvement.

\section{Materials and Methods}

\subsection{Construction of Citrus Intragenic Vector pUFCI}

Citrus-derived T-DNA-like regions were identified by BLAST searching the C. clementina genome sequence in Citrus Genome Database (http://www.citrusgenomedb. org/) using a T-DNA left border (LB) sequence (GTTTACACCACAATATATCCTGCCA) as a query [5]. Scaffold_89 (an assembled genomic DNA sequence), which harbors the first seven nucleotides of the LB sequence, prospective cloning sites, and the last seven nucleotides of a right border (RB) sequence, was selected. The rest of the LB sequence was obtained from Scaffold_2 and fused with the above T-DNA-like fragment through PCR amplification using primers CcF1 and CcR1 (Table 1). PCR products were directly ligated into the pGEM-T Easy vector and verified by sequencing. The plasmid DNA was digested with PstI and EcoRI to

Table 1. Sequences of the primers used in this study.

\begin{tabular}{|c|c|}
\hline Primer & Sequence (5’ - 3’) \\
\hline \multirow[t]{2}{*}{ CcF1 } & $\begin{array}{l}\text { GCTGCAGTTTACCCGCCAATATATCCTGTC } \\
\text { ATATTTTGAAACCAATATCAGAG }\end{array}$ \\
\hline & GGAATTCGGGCTAAGGCGGCAGTTCGGCG \\
\hline \multirow[t]{2}{*}{ CcR1 } & ATGGAGGTGGCAGGATATATTGTGGTGTA \\
\hline & AACGAGATGTTTGTTACTTATAGGAAACG \\
\hline VecF2 & $\begin{array}{l}\text { GCTGCAGCCG } \underline{G A T T C A T A C A G G C A G C C C ~} \\
\text { ATCAGTCC }\end{array}$ \\
\hline VecR1 & $\begin{array}{l}\text { GCTGCAGCTAAGAGAAAAGAGCGTTTATT } \\
\text { AGAATAATCG }\end{array}$ \\
\hline C-intra-F & CAAGAGGACAAGAGTCTATCC \\
\hline C-intra-R & TGAGGATGAAGACCTGAACG \\
\hline Ct-check-F & TTCAGGTCTTCATCCTCACG \\
\hline Ct-check-F2 & ATCGAGCACACACCATCATG \\
\hline Ct-check-R2 & AGCCTTAGGTTGTGACAGTG \\
\hline Ct-check-R3 & CGTTGGAGTGGAGTAATCAG \\
\hline Ct-check-R4 & TTTGTAAAGCGAGGAGCAGG \\
\hline Ct-check-R5 & TCTCTTGCCTCAGTTCAAGG \\
\hline IntraVecRBF1 & GTTTACCCGCCAATATATCCTG \\
\hline IntraVecRBF2 & TCATATTTTGAAACCAATATCAGAG \\
\hline IntraVecRBR & AGGTATCAGCATCTAACATCC \\
\hline $\mathrm{CtF} 2$ & ACGGAGTTCGGTTTGTTGTC \\
\hline CtR2 & AGCCTCAAGAGAGTTGCTAG \\
\hline $\mathrm{EF} 1 \alpha \mathrm{F}$ & AAGCCCATGGTTGTTGAGAC \\
\hline $\mathrm{EF} 1 \alpha \mathrm{R}$ & CAACAGCAAACTGGTGGAAG \\
\hline BamHI-BarF & GTGGATCCCCCGGGCTGCAGG \\
\hline SpeI-BarR & GACTAGTGGTCGACGGTATCGATAAGC \\
\hline
\end{tabular}

produce a sticky end fragment. The binary vector pCB302 was used as the template to generate the origin of replication and the nptIII expression cassette by PCR using primers VecF2 and VecR1 (Table 1) [8]. The PCR products were digested with PstI and EcoRI and ligated to the above sticky end citrus-derived T-DNA-like fragment to produce an intermediate vector pUFCI-1. To delete the duplicated SacI restriction site within the T-DNA-like region, pUFCI-1 was digested with SacI and self-ligated to further produce pUFCI-2. To avoid integration of vector backbone sequences beyond the LB into recipient plant genome during T-DNA transfer, a $\sim 3 \mathrm{~kb}$ fragment from C. clementina genome (scaffold_34) was inserted into pUFCI-2 between the LB and the vector backbone through the EcoRI site $[9,10]$. The direction of the added fragment was identified by PCR using primers Ct-check-F and Ct-check-F2 or Ct-check-R2 (Figure 1 and Table 1). The sequence of the $C$. clementina-derived T-DNA-like region and the buffering fragment outside of the LB in the final citrus intragenic vector, pUFCI, was confirmed by sequencing and shown in Figure 2. Three restriction sites, SpeI, SacI, and BamHI, in the T-DNAlike region can be utilized for gene cloning.

\section{2. pUFCI-Bar Plasmid Construction, Arabidopsis Transformation, and Selection}

A pair of primers BamHI-BarF and SpeI-BarR (Table 1) were used to amplify the entire expression cassette of the Bar gene from the binary vector pCB302 [8]. The PCR products were digested with BamHI and SpeI, and then ligated into the BamHI and SpeI sites of pUFCI. The resulting plasmid was introduced into the Agrobacterium tumefaciens strain GV3101(pMP90) by electroporation and transformed into the Arabidospsis thaliana (L.) Heynh. ecotype Columbia (Col-0) following the floral dip method [11]. Transformants were identified by spraying $\mathrm{T} 1$ seedlings with Basta.

\subsection{Citrus Transformation and Positive Regenerant Identification}

The empty vector was introduced into the $A$. tumefaciens strain EHA105 by electroporation and transformed into 'Duncan' grapefruit following the protocol described previously [12]. No selection pressure was exerted on explants. Regenerated shoots from individual transformation events were screened by PCR using primers C-intra-F and C-intra-R (Table 1). A characteristic 500 bp PCR product was used to identify positive regenerants. The LB and RB integration sites were mapped by PCR amplification using a set of primer pairs (Figure 1).

\section{Results and Discussion}

To facilitate development of intra-/cisgenic citrus cultivars, we have constructed an intragenic vector, pUFCI, 


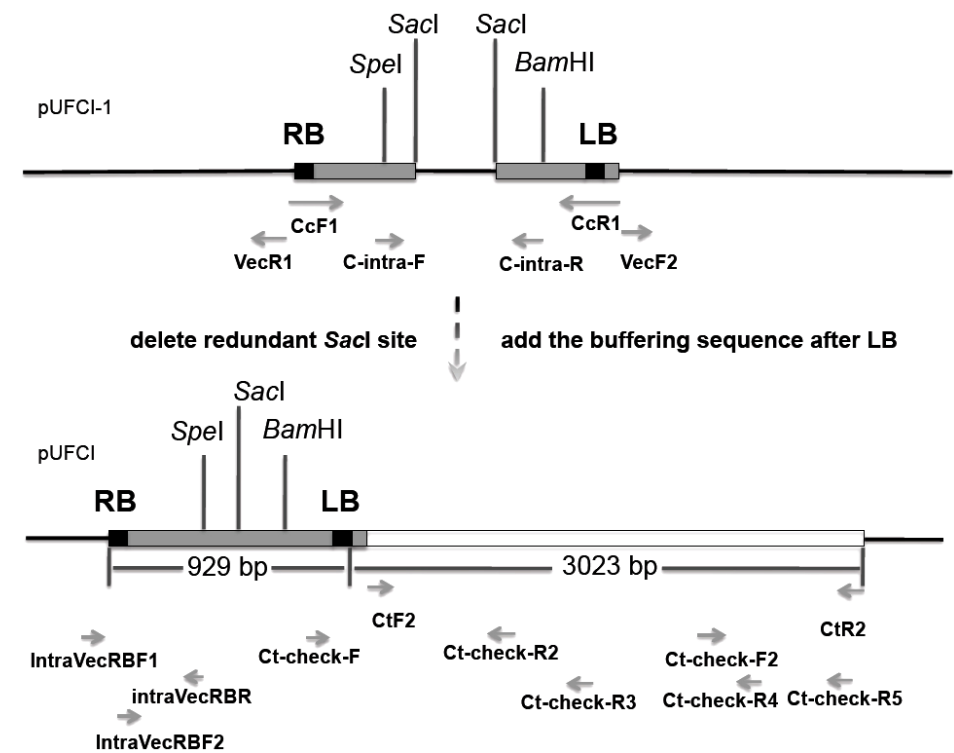

Figure 1. Schematic diagrams of the C. clementina-derived T-DNA-like regions and the positions of the PCR primers used in this study. LB: left border; RB: right border. The primer pairs CCF1/CcR1 and CtF2/CtR2 were used to amplify the T-DNA-like fragment and the GC rich buffering fragment from $C$. clementina, respectively. The vector backbone was generated by PCR amplification from the binary vector pCB302 using primers VecF2 and VecR1. The redundant SacI site in the T-DNA-like region was deleted. The primers $C$-intra-F and $C$-intra- $R$ were used for identification of positive regenerants. Ct-check-F, Ct-check-F2, and Ct-check-R2 were used to identify the orientation of the GC rich fragment. Ct-check-F, Ct-check-R2, Ct-check-R3, Ct-check-R4, and Ct-check-R5 were used to map the integration site outside of the LB. IntraVecRBR, IntraVecF1, and IntraVecF2 were used to check the integration site of the RB. Three restriction sites, SpeI, SacI, and BamHI, between the $R B$ and $L B$ are available for gene cloning.

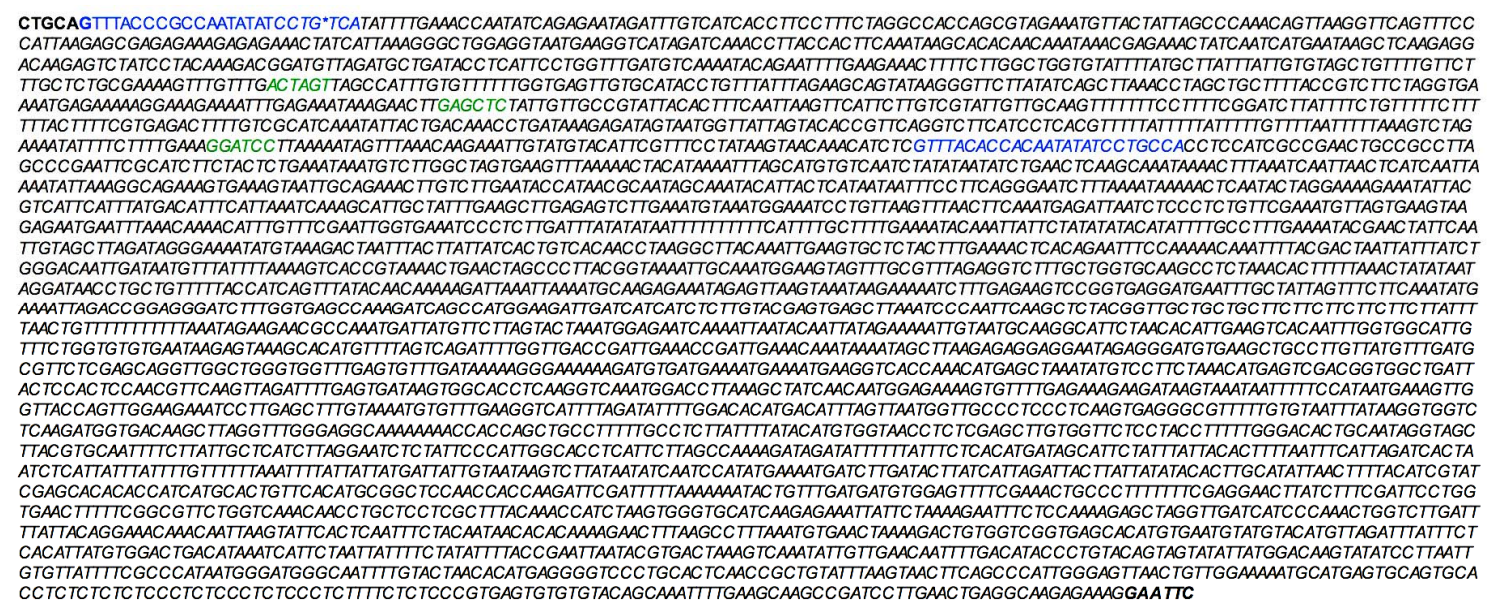

Figure 2. Sequence of the T-DNA-like region and the buffering fragment in pUFCI. All nucleotides in the T-DNA-like region and the $\sim 3 \mathrm{~kb}$ buffering fragment outside of the left border were assembled from $C$. clementina. The nucleotides from the $C$. clementina genome are italicized, with the T-DNA borders in blue and the three unique restriction sites (SpeI, SacI, and BamHI) in green. The asterisk ( $\left(^{*}\right)$ in the right border indicates the site of T-strand initiation for T-DNA transfer to plants. The nucleotide fragment shown in the figure was linked to the backbone of the binary vector pCB302 using the PstI and EcoRI sites (in bold at each end).

which bears a T-DNA-like region with functional equivalents of T-DNA border sequences from C. celementina (Figure 3). Within this region three restriction sites (SpeI, $S a c I$, and BamHI) can be used for cloning native genes from sexually compatible citrus species.

In the new citrus intragenic vector, we designed a sim- ple PCR amplification strategy for identification of positive regenerants using the size difference caused by deletion of the duplicated SacI restriction site within the T-DNA-like region. For the empty vector, positive regenerants gave a specific $\sim 500$ bp fragment together with a $\sim 1 \mathrm{~kb}$ fragment amplified from the recipient "Duncan” 


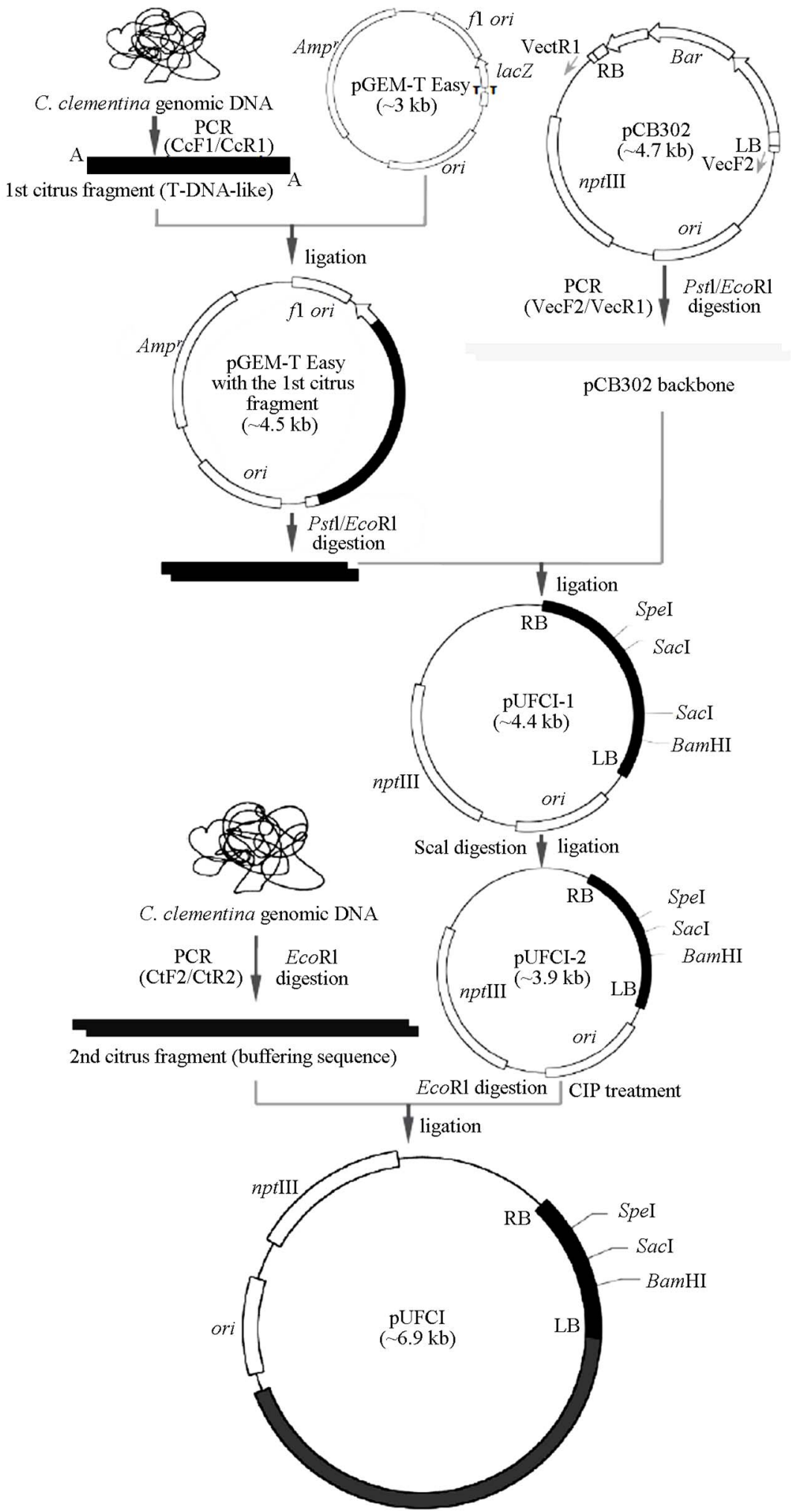

Figure 3. Construction of the citrus intragenic vector pUFCI. LB: left border, RB: right border. Explanation is detailed in 2.1.

grapefruit genome (Figure 4(B)). When a gene cassette of interest is ligated into pUFCI and introduced into re- cepient citrus genome, positive regenerants can be easily identified through PCR amplification using a gene specific 

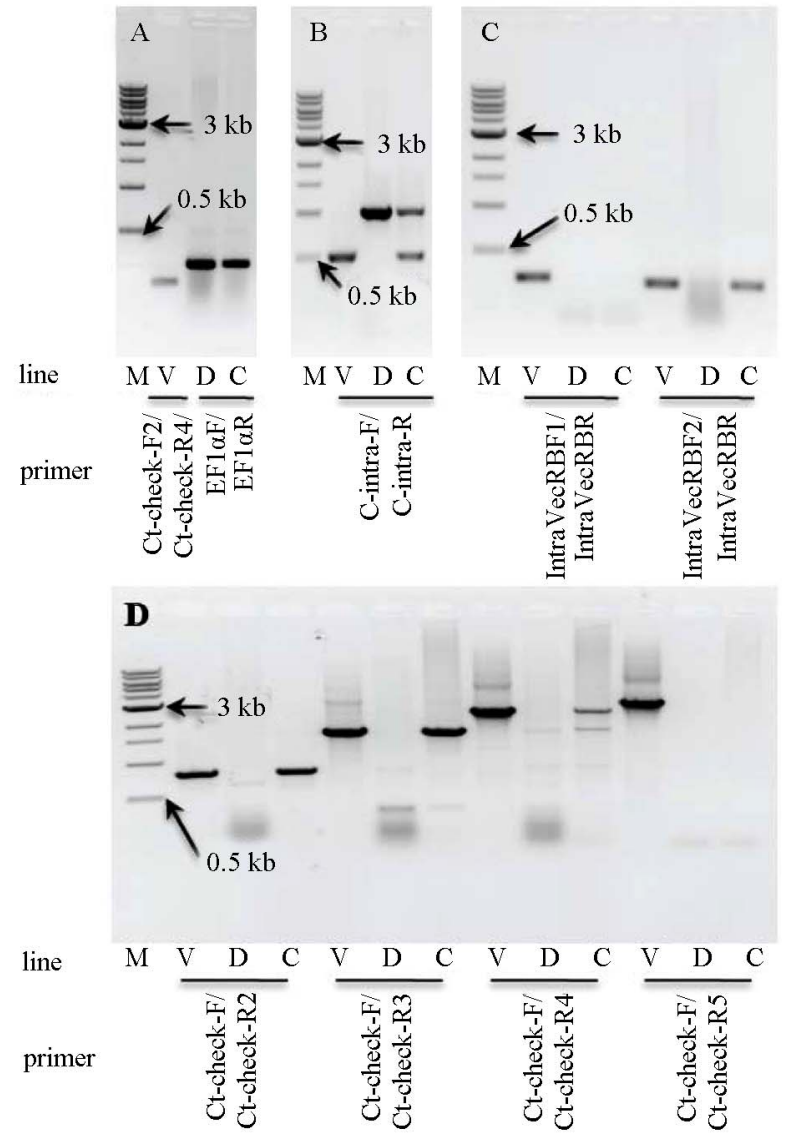

Figure 4. Characterization of a representative intra-/cisgenic citrus plant generated with the empty pUFCI vector. (A) PCR confirmation of the presence of the respective DNA in the three DNA samples used in (B)-(D); The pUFCI plasmid DNA (V) was amplified with the primers Ct-check-F2 and Ct-check-R4, yielding a 185 bp fragment. The wild-type "Duncan" grapefruit DNA (D) and the cisgenic "Duncan" grapefruit DNA (C) were amplified with primers for the citrus EF1 $\alpha$ gene, producing a $\sim 300$ bp fragment. M: 1 kb DNA marker; (B) A characteristic $\sim 500$ bp fragment was amplified from V and C, but not from $D$, whereas a $\sim 1 \mathrm{~kb}$ fragment was amplified from $D$ and $C$, but not from $V$, confirming the integration of the pUFCI T-DNA-like fragment into the recipient "Duncan" grapefruit genome; (C) No vector backbone sequences adjacent to the RB were integrated in the intra-/cisgenic plant. The forward primers IntraVecRBF1 and IntraVecRBF2 anneal to the first 22 nucleotides of the RB and the citrus-derived sequence including the last three nucleotides of the RB, respectively, and the reverse primer IntraVecRBR is located inside the T-DNA-like region. PCR products were amplified with IntraVecRBF2 and IntraVecRBR but not with IntraVecRBF1 and IntraVecRBR from $C$, indicating that the T-DNA transfer in the intra-/cisgenic plant initiated in the RB; (D) No vector backbone sequences adjacent to the buffering sequence on the $L B$ side were integrated into the intra-/cisgenic plant. $\mathrm{Ct}$-check-F is the forward primer located in the T-DNA-like region, and Ct-check-R2, Ct-check-R3, Ct-check-R4, and Ct-check-R5 are reverse primers located at different positions in the buffering sequence. PCR products were amplified from $\mathrm{V}$ but not from $\mathrm{C}$ with the primers $\mathrm{Ct}$-check-F1 and $\mathrm{Ct}$-check-R5, indicating that the T-DNA transfer in the intra-/cisgenic plant ended between primers Ct-check-R4 and Ct-check-R5.

primer in combination with a primer annealing to the T-DNA-like region. Transferring and integration of T-DNA into the plant genome by Agrobacteria initiate from the RB and end at the LB. However, integration of binary vector backbone sequences especially the sequence outside of the LB in transgenic plants is a common phenomenon [5]. To decrease the possibility of the presence of non-citrus sequences in the regenerants, we added a $\sim 3 \mathrm{~kb}$ C. clementina-originated DNA fragment outside of the LB to serve as a buffering sequence. It permits a tolerance towards truncations beyond the LB without interfering with the concept of gene transfer without “foreign DNA". As expected, integration of the pUFCI T-DNA-like region into the 'Duncan' grapefruit genome started at the RB without integration of any backbone sequences (Figure $\mathbf{4}(\mathbf{C})$ ). Our characterization of a representative intra-/cisgenic citrus plant showed the presence of DNA sequences beyond the LB. However, it stopped before the end of the buffering sequence (Figure 4(D)). Therefore, no bacteria-derived backbone DNA was introduced into the regenerated citrus plant. These results demonstrate the possibility of using pUFCI to 


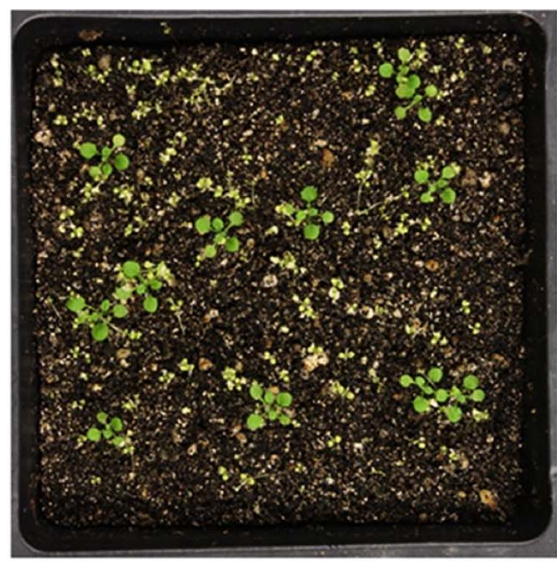

pUFCI

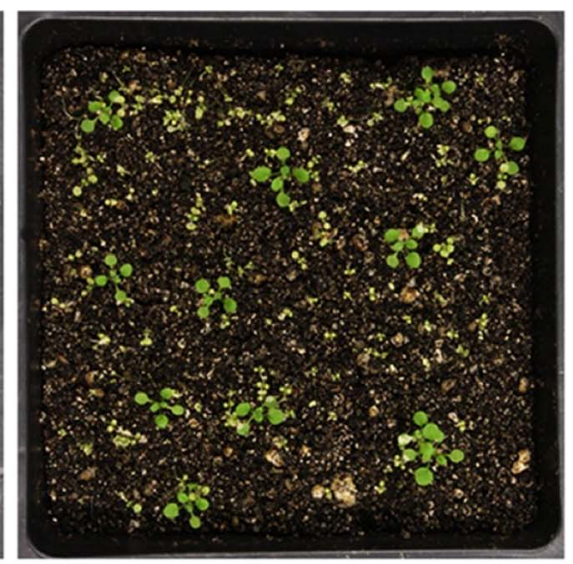

pCB302

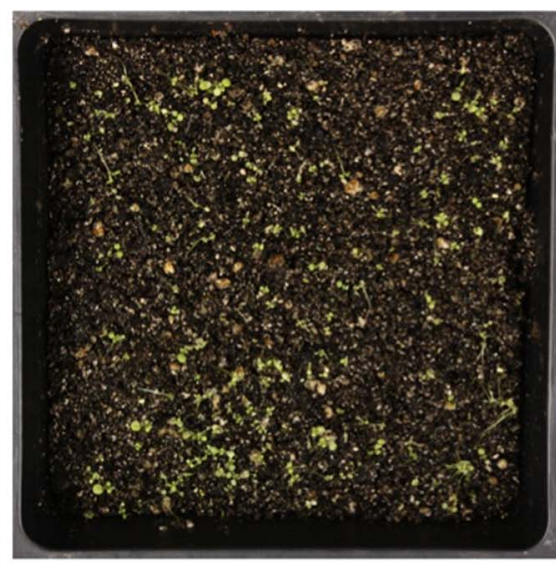

Untransformed

Figure 5. The pUFCI vector mediates efficient genetic transformation in Arabidopsis. A. thaliana ecotype Col-0 plants were transformed with Agrobacteria carrying the plasmid pUFCI-Bar or the T-DNA binary vector pCB302 and the T1 seedlings were sprayed with Basta. Untransformed Col-0 plants were used as negative controls. The average transformation efficiencies were calculated from eight replicated experiments. Green seedlings are transgenic plants

generate “foreign DNA-free” intra-/cisgenic citrus plants.

To test the transformation efficiency of the intragenic vector, we cloned the expression cassette of the Bar gene into pUFCI and transformed Arabidopsis with the resulting plasmid pUFCI-Bar. Basta screening gave an average transformation efficiency of $\sim 3 \%$ in the A. thaliana ecotype Col-0, which is comparable to the efficiency of pCB302 and other widely used binary vectors for Arabidopsis transformation (Figure 5) [8]. These results indicate that the $C$. clementina-originated sequences in pUFCI do not contain silencing components. Furthermore, we tested transformation efficiency of pUFCI in citrus. An average transformation efficiency of $\sim 0.67 \%$ was achieved in our three independent transformation experiments. Similar levels of transformation efficiency were observed in the production of intra-/cisgenic apple plants [13]. Based on our current transformation protocol, the efficiency is acceptable for generating "foreign DNA-free” intra-/cisgenic plants in citrus [7,14,15].

\section{Conclusion}

pUFCI is a versatile intragenic vector that can generate intra-/cisgenic citrus and transgenic Arabidopsis with high frequencies. Identification of positive intra-/cisgenic citrus regenerants can be accomplished by simple PCR analysis. Considering the current citrus transformation protocol and the availability of the citrus genome sequences for candidate gene identification, developing intra-/cisgenic citrus plants with genes of interest using pUFCI is applicable for future citrus genetic improvement.

\section{Acknowledgements}

This research was supported by grants from the Citrus
Research and Development Foundation and National Science Foundation (IOS-0842716) awarded to ZM.

\section{REFERENCES}

[1] A. J. Conner, T. R. Glare and J. P. Nap, "The Release of Genetically Modified Crops into the Environment: II. Overview of Ecological Risk Assessment,” Plant Journal, Vol. 33, No. 1, 2003, pp. 19-46. http://dx.doi.org/10.1046/j.0960-7412.2002.001607.x

[2] R. B. Flavell, E. Dart, R. L. Fuchs and R. T. Fraley, "Selectable Marker Genes: Safe for Plants?” Nature Biotechnology, Vol. 10, No. 2, 1992, pp. 141-144. http://dx.doi.org/10.1038/nbt0292-141

[3] B. Darbani, A. Eimanifar, C. N. Stewart and W. N. Camargo, "Methods to Produce Marker-Free Transgenic Plants,” Biotechnology Journal, Vol. 2, No. 1, 2007, pp. 83-90. http://dx.doi.org/10.1002/biot.200600182

[4] M. Malnoy, E. E. Boresjza-Wysocka, J. L. Norelli, M. A. Flaishman, D. Gidoni and H. S. Aldwinckle, "Genetic Transformation of Apple (Malus x domestica) without Use of a Selectable Marker Gene,” Tree Genetics \& Genomes, Vol. 6, No. 3, 2010, pp. 423-433. http://dx.doi.org/10.1007/s11295-009-0260-7

[5] A. J. Conner, P. J. Barrell, S. J. Baldwin, A. S. Lokerse, P. A. Cooper, A. K. Erasmusn, J. P. Nap and J. M. E. Jacobs, "Intragenic Vectors for Gene Transfer without Foreign DNA,” Euphytica, Vol. 154, No. 3, 2007, pp. 341-353. http://dx.doi.org/10.1007/s10681-006-9316-z

[6] C. M. Rommens, M. A. Haring, K. Swords, H. V. Davies and W. R. Belknap, "The Intragenic Approach as a New Extension to Traditional Plant Breeding," Trends in Plant Science, Vol. 12, No. 9, 2007, pp. 397-403.

http://dx.doi.org/10.1016/j.tplants.2007.08.001

[7] A. Ballester, M. Cervera and L. Peña, "Selective MarkerFree Transgenic Orange Plants Recovered under NonSelective Conditions and through PCR Analysis of All 
Regenerants,” Plant Cell, Tissue and Organ Culture, Vol. 102, No. 3, 2010, pp. 329-336.

http://dx.doi.org/10.1007/s11240-010-9737-1

[8] C. Xiang, P. Han, I. Lutziger, K. Wang and D. J. Oliver, "A Mini Binary Vector Series for Plant Transformation," Plant Molecular Biology, Vol. 40, No. 4, 1999, pp. 711717. http://dx.doi.org/10.1023/A:1006201910593

[9] S. B. Gelvin, "Agrobacterium-Mediated Plant Transformation: The Biology behind the 'Gene-Jockeying' Tool," Microbiology and Molecular Biology Reviews, Vol. 67, No. 1, 2003, pp. 16-37. http://dx.doi.org/10.1128/MMBR.67.1.16-37.2003

[10] C. M. Rommens, O. Bougri, H. Yan, J. M. Humara, J. Owen, K. Swords and J. Ye, "Plant-Derived Transfer DNAs,” Plant Physiology, Vol. 139, No. 3, 2005, pp. 1338-1349. http://dx.doi.org/10.1104/pp.105.068692

[11] S. J. Clough and A. F. Bent, "Floral Dip: A Simplified Method for Agrobacterium-Mediated Transformation of Arabidopsis thaliana," Plant Journal, Vol. 16, No. 6, 1998, pp. 735-743. http://dx.doi.org/10.1046/j.1365-313x.1998.00343.x

[12] V. Orbovic and J. W. Grosser, "Citrus: Sweet Orange
(Citrus sinesis L. Osbeck 'Valencia') and Carrizo Citrange (Citrus sinesis (L.) Osbeck x Poncirus trifoliate (L.) Raf.)," In: K. Wang, Eds., Agrobacterium ProtocolMethods in Molecular Biology, Humana Press, New York, 2006, pp. 177-189.

[13] T. Vanblaere, I. Szankowski, J. Schaart, H. Schouten, H. Flachowsky, G. A. Broggini and C. Gessler, "The Development of a Cisgenic Apple Plant," Journal of Biotechnology, Vol. 154, No. 4, 2011, pp. 304-311. http://dx.doi.org/10.1016/j.jbiotec.2011.05.013

[14] A. Ballester, M. Cervera and L. Peña, "Evaluation of Selection Strategies Alternative to nptII in Genetic Transformation of Citrus,” Plant Cell Report, Vol. 27, No. 6, 2008, pp. 1005-1015. http://dx.doi.org/10.1007/s00299-008-0523-z

[15] M. G. C. Costa, W. C. Otoni and G. A. Moore, "An Evaluation of Factors Affecting the Efficiency of Agrobacterium-Mediated Transformation of Citrus paradisi (Macf.) and Production of Transgenic Plants Containing Carotenoid Biosynthetic Genes,” Plant Cell Report, Vol. 21, No. 4, 2002, pp. 365-373. http://dx.doi.org/10.1007/s00299-002-0533-1 